\title{
How to handle the delayed or the missed dose of edoxaban in patients with non-valvular atrial fibrillation: model-informed remedial strategy
}

\author{
Yi-wei Yin ${ }^{1}$, Xiaoqin $\mathrm{Liu}^{2}$, Jiaqin $\mathrm{Gu}^{1}$, Ziran $\mathrm{Li}^{2}$, and Zheng Jiao ${ }^{1}$ \\ ${ }^{1}$ Shanghai Jiao Tong University Affiliated Chest Hospital \\ ${ }^{2}$ Huashan Hospital Fudan University
}

November 26, 2021

\begin{abstract}
Aim: Edoxaban is a non-vitamin K antagonist oral anticoagulant (NOAC) widely used for long-term stroke prevention in patients with non-valvular atrial fibrillation (NVAF). Adherence to NOACs is unsatisfactory and decreases over time. The aim of this study was to explore appropriated remedial dosing regimens for non-adherent edoxaban-treated NVAF patients through the Monte Carlo simulation. Methods: Six proposed regimens were compared with the recommendations in the European Heart Rhythm Association (EHRA) guide regarding the trough total deviation time considering both edoxaban plasma concentration and inactive factor Xa activity. Monte Carlo simulations were performed using RxODE based on the published population pharmacokinetics/pharmacodynamics model. Results: The proposed remedial strategies were different from EHRA recommendations and were related to delay duration. The missed dose can be taken immediately if the delay time is within $11 \mathrm{~h}$. When the delay period is between 12 and $19 \mathrm{~h}$, a half dose followed by a regular dosing schedule is recommended. When the delay time exceeds $19 \mathrm{~h}$, a full dose followed by a half dose is preferred compared to that recommended in the EHRA guide. Our proposed strategies resulted in a shorter total deviation time than EHRA guide. Conclusion: PK/PD modelling and simulation are effective in developing and evaluating the remedial strategies of edoxaban, which could help maximize its therapeutic effect.
\end{abstract}

\section{Introduction}

Medication nonadherence has long been a persistent and yet unsolved challenge ${ }^{1}$. Adherence is important to achieve the desired therapeutic outcome, especially for drugs associated with a quick loss of therapeutic effect when non-adherence occurs, including non-vitamin $\mathrm{K}$ oral anticoagulants (NOACs) ${ }^{2}$. It is concerning that patients implement variable and potentially inappropriate remedial strategies in real life ${ }^{3}$.

Edoxaban is a commonly used non-vitamin $\mathrm{K}$ oral anticoagulant (NOAC). It has been approved for stroke prevention in patients with nonvalvular atrial fibrillation (NVAF), as it selectively inhibits active clotting factor $\mathrm{X}(\mathrm{FXa})^{4}$. Edoxaban reaches its maximum plasma concentration within 1-2 h after oral administration with a volume distribution of $107 \mathrm{~L}$ in adults. It has a terminal elimination half-life of approximately 10-14 h with equal renal and non-renal clearance. Additionally, Edoxaban is a substrate of P-glycoprotein (P-gp) and hence, should not be used with strong P-gp inducers (e.g., rifampin) or inhibitors ${ }^{5}$. According to previous research, dose adjustment can be applied to underweight patients, patients with impaired renal function, and patients concomitantly using a P-gp inhibitor ${ }^{6}$.

Edoxaban is a drug with fast-on and fast-off effects, similar to other NOACs ${ }^{7}$. The anticoagulant effect of edoxaban can disappear at $12-24 \mathrm{~h}$ after discontinuation ${ }^{8}$. Research has shown that adherence to NOACs is unsatisfactory and decreases over time. In patients with NVAF, adherence to NOACs was $<70 \%$ after 12 
months of treatment ${ }^{9}$. Low adherence to NOACs is associated with an increased risk of stroke and death. According to a retrospective study enrolled $>60,000$ patients from the United States, non-adherent atrial fibrillation patients with $\mathrm{CHA}_{2} \mathrm{DS}_{2}$-VASc score [?] 2 were at a higher risk of stroke comparing to adherent patients ${ }^{10}$. Due to the undesired outcomes of non-adherence, the American Heart Association and European Heart Rhythm Association (EHRA) emphasise the importance of a strict adherence to NOACs in atrial fibrillation patients ${ }^{11,12}$. However, the proportion of non-adherent patients is still high, and developeing approaches to help non-adherent patient to restore anticoagulant effect as soon as possible is essential. Several generic recommendations are available for edoxaban. According to the package inserts approved by the Food and Drug Administration (FDA), the patient need to, "If a dose of edoxaban is missed, the dose should be taken as soon as possible on the same day. Dosing should resume the next day according to the normal dosing schedule. The dose should not be doubled to make up for a missed dose"13. EHRA recommends, "for NOACs with a once-a-day dosing regimen, a delayed dose can be taken up until $12 \mathrm{~h}$ after the scheduled intake. After this time point, the dose should be skipped, and the next scheduled dose should be taken" 12 . Similar recommendations were found in the Electronic Medicines Compendium in the United Kingdom ${ }^{14}$. However, none of the available recommendation is based on clinical evidence or validated. Additionally, it is unclear whether these recommendations can be applied to specific subpopulations, such as underweight patients, patients with renal impairment, and patients that use P-gp inhibitors.

Due to ethical reasons, it is difficult to conduct clinical studies to explore and evaluate remedial strategies. Population pharmacokinetic/pharmacodynamic (PK/PD) modelling and simulations provide a practical approach ${ }^{15}$. This method has also been successfully applied to antiepileptic, antipsychotic, and immunosuppressive agents ${ }^{16-19}$. In our previous study, we have successfully developed model-informed remedial strategies for rivaroxaban based on PK/PD modelling and found that the EHRA guide may not provide best solution for dealing with delayed or missed dose ${ }^{20}$. In this study, we aimed to assess the effect of nonadherence and explore appropriate remedial strategies through modelling and simulation in patients with NVAF treated with edoxaban.

\section{Methods}

\subsection{Patients and dosage regimen}

We set two typical patients with normal and impaired renal function (creatinine clearance $[\mathrm{CrCl}]$ of 80 and $40 \mathrm{ml} / \mathrm{min}$, respectively). Detailed demographic and dosage information are listed in Table 1 . We considered the patients age to be 75 years-old based on the reported median age of $\mathrm{NVAF}^{21}$. Typical patients and related dose regimens for simulation were developed based on the consensus of US FDA package insert, European Medicines Agency leaflet, and European Society of Cardiology 2020 guideline. The standard dose of edoxaban for NVAF is $60 \mathrm{mg}$ daily. Dose needs to be reduced to $30 \mathrm{mg}$ daily in patients with $\mathrm{CrCl}$ between 15 and $50 \mathrm{~mL} / \mathrm{min}^{13,14,22}$.

\subsection{Remedial strategy assessment}

Various non-adherence dose scenarios within one dose interval $(24 \mathrm{~h})$ were developed and assessed for each typical patient, as listed in Table 1. A total of 24 scenarios were simulated with one dose interval delayed from 1 to $24 \mathrm{~h}$ after the regular time at hourly intervals (Figure 1).

\subsubsection{Population PK/PD model and simulation}

A previously published population PK/PD model of edoxaban was used in our study ${ }^{23}$. . To the best of our knowledge, this is the only published combined PK and PD model. This model was developed based on phase I and phase II studies. A total of 1,624 healthy subjects and NVAF patients were enrolled in the PK analysis, and a total of 585 NVAF patients were enrolled in the PD analysis. PK was described by a two-compartment model with first-order absorption with lag time and first-order elimination. The intrinsic FXa activity (iFXa) was selected as a PD marker and was modelled using a dynamic binding and equilibrium model. The PD data were modeled using dynamic binding model and equilibrium model. A summary of the demographic information of the modelling population is presented in Supplementary Table 1. The PK/PD 
model and all parameter estimates employed in the Monte Carlo simulation are listed in Table 2.

Monte Carlo simulations were performed with RXODE (version 0.9.1-8) in R (version 3.6.1) ${ }^{24}$. Under each non-adherence scenario, one thousand virtual patients were simulated to obtain both the PK and PD profiles. The patients were assumed to reach a steady state before the delayed dose. Additionally, it was assumed that the current dose was sufficient to produce the desired anticoagulation effect without undesirable adverse effects.

\subsubsection{Remedial dosing strategies}

Six remedial strategies were developed considering their clinical feasibility and our previous studies ${ }^{20}$. Remedial strategies are the action patient will take when they remember their missed dose. Remedial strategies refer to actions that patients will take when they remember their missed dose. As edoxaban tablets are divisible, we included a half dose in our remedial strategy to provide a more precise approach. We tested both the current recommendations from the EHRA guide and our developed remedial strategies. Our remedial strategy is similar to our previous study in rivaroxaban, which are shown in Figure 2 and detailed below ${ }^{20}$ :

Strategy A: skip the full delayed dose and take the next dose as schedule.

Strategy B: take the full delayed dose immediately and take the next dose as schedule.

Strategy C: take a half delayed dose immediately and take the next dose as schedule

Strategy D: take a full delayed dose immediately followed by a half dose at the next scheduled time, and then resume the regular dosing regimen.

Strategy E: take one and a half delayed doses immediately, skip the next scheduled dose, and then resume the regular dosing regimen.

Strategy F: take a double of delayed dose, skip the next scheduled dose, and then resume the regular dosing regimen.

EHRA guide: take the delayed dose if less than $12 \mathrm{~h}$ late; otherwise, skip the delayed or missed dose and administer the next dose at the scheduled time.

\subsubsection{Index for evaluation of remedial dosing strategies}

Currently, there is no well-defined therapeutic range for edoxaban concentration. Therefore, the concept of "on-therapy range" was employed. It was built on the assumption that patients were at therapeutic goals when they reached full adherence. In our study, we defined the on-therapy range as between the $5^{\text {th }}$ percentile of trough and the $95^{\text {th }}$ percentile of peak for both concentration and iFXa based on previous studies ${ }^{25-27}$.

Non-adherence scenarios described previously were simulated for both PK and PD, and the percentages of individuals outside the on-therapy range were estimated. Moreover, the remedial strategies listed above were assessed for each non-adherence scenario. The total deviation time for each strategy was defined as the sum of the deviation time outside the on-therapy range of edoxaban concentration or iFXa and was calculated for each strategy.

The goal of the remedial strategy was to help patients minimise out-of-range duration. The strategy with the least total deviation time was considered optimal for each non-adherence scenario. Meanwhile, two remedial strategies were considered equivalent if the difference in the total deviation time was less than $1 \mathrm{~h}$ for the same non-adherence scenario.

\subsubsection{Sensitivity analysis}

Sensitivity analysis was applied to identify parameters that potentially impacted model outcome and their contribution to the overall variability. Sensitivity was assessed by the change in clearance (CL/F, 50-100\%), $\mathrm{CrCl}(20-80 \mathrm{ml} / \mathrm{min})$, and body weight $(40-100 \mathrm{~kg})$. The change in CL/F was assumed to reflect the impact of co-administration with a certain P-gp inhibitor (e.g. dronedarone, cyclosporine, and erythromycin), as 
concomitant use increased edoxaban exposure by $40 \%$ to $84.5 \%$ according to a previous study ${ }^{28}$. The detailed demographic and dosage information for sensitivity analysis are listed in supplementary Table 2.

Irregularly dosed intervals of 22-26 h (09:00, 07:00, and 09:00) and 26-22 h (09:00, 11:00, and 09:00) were tested considering that patients might take edoxaban at slightly different times every day. To ensure equivalence, the criterion used to evaluate the equality of different strategies was tested from 1-3 h. Sensitivity analysis was performed when the dose was delayed for $6,12,18$, and $24 \mathrm{~h}$.

\section{Results}

\subsection{Effect of non-adherence on PK/PD of edoxaban}

According to the PK/PD simulation, the effect of non-adherence was dependent on the delay time, as the risk of patients outside the on-therapy range increased with increasing delay time. Under the same non-adherent scenario, the percentage of patients out of range based on iFXa was slightly lower than the percentage of patients out of ranged based on edoxaban concentration (Figure 3).

For example, based on edoxaban concentration, $16.3 \%$ and $39.1 \%$ of patients with normal renal function who took $60 \mathrm{mg}$ of edoxaban daily were out of range when delayed for 12 and $24 \mathrm{~h}$, respectively. Similar results were observed in patients with impaired renal function taking $30 \mathrm{mg}$ of edoxaban daily ( $15.3 \%$ and $36.9 \%$ when delayed for 12 and $24 \mathrm{~h}$, respectively). Moreover, based on iFXa activity, $8.7 \%$ and $24.1 \%$ of patients with normal renal function were out-of-range when delayed for 12 and $24 \mathrm{~h}$, respectively, and $7.8 \%$ and $24.1 \%$ of patients with impaired renal function were out of range when delayed for 12 and $24 \mathrm{~h}$.

\subsection{Remedial strategies}

The remedial dose strategies for two typical patients based on edoxaban concentration and iFXa activity are summarized in Table 3. Figure 4 showed the edoxaban concentration and iFXa profile for patients (aged 75 years, $\mathrm{CrCl} 80 \mathrm{~mL} / \mathrm{min}$, TBW 80kg, receiving $60 \mathrm{mg} \mathrm{q} 24 \mathrm{~h}$ ) with full adherent and with a proposed remedial strategies while delay $10 \mathrm{~h}$. Two typical patients had a similar optimised remedial regimen. According to our result, a full dose of edoxaban could be taken immediately up to $11 \mathrm{~h}$ after a delayed dose for both typical patients. When the delay time was between 12 and $19 \mathrm{~h}$, patients were recommended to take a half dose and then resume the normal dosing schedule. When the delay exceeds $19 \mathrm{~h}$, a full dose followed by a half dose was preferred.

Recommendations based on PK and iFXa are similar in patients with normal and impaired renal function.

Based on our research, the full dose needs to be taken immediately in the first $11 \mathrm{~h}$ after dose delay, as recommended by the EHRA. However, the EHRA recommends taking the full dose at a delay of $12 \mathrm{~h}$ and skip the missed dose after a $12 \mathrm{~h}$ delay, which yields a longer total deviation time compared to our recommendations. For example, in $80 \mathrm{~kg}$ patients with normal renal function, when the dose is delayed for $14 \mathrm{~h}$, the deviation time for EHRA recommendation is $73 \%$ longer than our recommendation $(27.4 \mathrm{~h}$ versus $15.8 \mathrm{~h}$ ) based on PK and $83 \%$ longer (27.2 versus $14.8 \mathrm{~h}$ ) based on PD. A comparison between the EHRA and our proposed remedial strategies is shown in Figure 5. The detailed deviation time information for different strategies is listed in Supplementary table 3.

\subsection{Sensitivity analysis}

The change in deviation time did not significantly alter the remedial strategies when CL/F (50-100\%), $\mathrm{CrCl}$ (20-80 $\mathrm{ml} / \mathrm{min})$, and body weight $(40-100 \mathrm{~kg}$ ) were taken into account. Moreover, the change in criterion for evaluating the equality from $1-3 \mathrm{~h}$ did not significantly change the remedial dosing recommendations.

\section{Discussion}

To the best of our knowledge, this is the first study to comprehensively develop and evaluate different remedial strategies during non-adherence events in edoxaban treated patients with NVAF. We developed an optimised remedial strategy based on non-adherence scenarios using PK and PD population modelling. Unlike previous studies that focused on PK, our study considered both PK and PD ${ }^{17-19}$. iFXa percentage 
was used as a PD marker in our study, which provides a closer relationship to the clinical outcome than exposure and can help to achieve a more appropriate remedial strategy.

In our study, we used the on-therapy range to overcome the challenge of lacking a defined therapeutic range. The on-therapy range provides a dynamic goal based on the patient's individual demographic and indicated dosage regimen, which mimics the real-life situation. Additionally, we performed sensitivity analysis to extend our recommendation to patients receiving P-gp inhibitors and/or with low body weight.

According to our PK/PD modelling and simulation, the EHRA recommendation is not optimal and can only be applied when the delay [?] 11 hours. These results are similar to those of our previous study, showing that EHRA also does not provide optimal recommendations for rivaroxaban missing $\operatorname{dose}^{20}$. Our study shows that from the PK/PD perspective, there is potential room for improvement in the remedial strategy in current practice. The current practice has missed the opportunity to help patients address their missed or delayed drug in a more appropriate manner.

In practice, patients can have different risks of bleeding and thrombosis, which can be assessed through scoring systems such as HAS-BLED or $\mathrm{CHA}_{2} \mathrm{DS}_{2}$-VASc for bleeding and stroke, respectively ${ }^{29,}{ }^{30}$. Other risk factors, including concomitant drug use, can also alter a patient's risk of bleeding and thrombosis. Optimal remedial strategies can be modified based on the patient's risk of bleeding and thrombosis. It is necessary that healthcare providers balance patients' risk of bleeding and thrombosis by considering the deviation time above and below the on-therapy range.

Our study has several limitations. First, the results of our study represent the values from the modelling population and may not be extrapolated to patients who do not match the modelling population, especially in patients who take edoxaban for other indications, including venous thromboembolism. Second, the results of our study were not evaluated in real-world patients. However, due to ethical reasons, PK/PD modelling and simulation is the preferred approach for developing and testing remedial strategies in the target population.

\section{Conclusion}

Our study assessed the effects of non-adherence to edoxaban on PK and PD. We developed an appropriate remedial dosing strategy for NVAF patients based on the populational PK/PD model. Our simulation has shown that current EHRA recommendations may not be the optimal remedial dosing for patients with delayed or missed doses. Model-informed simulation is an accessible method for effectively addressing the remedial dosing strategy. In practice, clinicians can use our strategy and balance patients' thrombosis and bleeding risk to determine the optimal remedial strategy.

\section{Declaration}

\section{Conflict of interest}

The Authors declare that there is no conflict of interest.

\section{Funding}

This work was supported by Young Researcher Development Supporting Program of Shanghai Chest Hospital, Shanghai Jiao Tong University

\section{Data availability statement}

All data generated or analysed during this study are included in this published article and its supplementary information files.

\section{Author contributions}

Yi-wei Yin : Methodology, Formal analysis, Writing-original draft, Writing-review \& editing, Xiao-qin Liu : Writing-original draft, Writing-review \& editing, Visualization.Jia-qin Gu : Writing-original draft, Formal analysis, Writing-review \& editing, Zi-ran Li: Formal analysis, Data curation, Visualization. Zheng Jiao 
: Conceptualization, Methodology, Writing-original draft, Writing-review \& editing, Supervision, Project administration.

\section{Reference}

1. Kleinsinger F. The Unmet Challenge of Medication Nonadherence. Perm J. 2018;22:18-033

2. Hughes DA. Medication adherence-Key considerations for clinical pharmacologists. Br J Clin Pharmacol. 2020;86(4):628-9.

3. Albassam A, Hughes DA. What should patients do if they miss a dose? A systematic review of patient information leaflets and summaries of product characteristics. Eur J Clin Pharmacol. 2021;77(2):251-60.

4. Srinivasan S, Ajmal M, Pecci C, Lassar T. Edoxaban in Cardiovascular Disease Management: Review. Br J Clin Pharmacol. 2021. doi: 10.1111/bcp.15026.

5. Parasrampuria DA, Truitt KE. Pharmacokinetics and Pharmacodynamics of Edoxaban, a Non-Vitamin K Antagonist Oral Anticoagulant that Inhibits Clotting Factor Xa. Clin Pharmacokinet. 2016;55(6):641-55.

6. Giugliano RP, Ruff CT, Braunwald E, Murphy SA, Wiviott SD, Halperin JL, et al. Edoxaban versus warfarin in patients with atrial fibrillation. N Engl J Med. 2013;369(22):2093-104.

7. Moll S, Berkowitz JN, Miars CW. Elite athletes and anticoagulant therapy: an intermittent dosing strategy. Hematology Am Soc Hematol Educ Program. 2018;2018(1):412-7.

8. Deshpande CG, Kogut S, Laforge R, Willey C. Impact of medication adherence on risk of ischemic stroke, major bleeding and deep vein thrombosis in atrial fibrillation patients using novel oral anticoagulants. Curr Med Res Opin. 2018;34(7):1285-92.

9. Pham PN, Brown JD. Real-world adherence for direct oral anticoagulants in a newly diagnosed atrial fibrillation cohort: does the dosing interval matter? BMC Cardiovasc Disord. 2019;19(1):64.

10. Yao X, Abraham NS, Alexander GC, Crown W, Montori VM, Sangaralingham LR, et al. Effect of Adherence to Oral Anticoagulants on Risk of Stroke and Major Bleeding Among Patients With Atrial Fibrillation. J Am Heart Assoc. 2016;5(2): e003074

11. Nishimura RA, Otto CM, Bonow RO, Carabello BA, Erwin JP, 3rd, Guyton RA, et al. 2014 AHA/ACC Guideline for the Management of Patients With Valvular Heart Disease: executive summary: a report of the American College of Cardiology/American Heart Association Task Force on Practice Guidelines. Circulation. 2014;129(23):2440-92.

12. Steffel J, Verhamme P, Potpara TS, Albaladejo P, Antz M, Desteghe L, et al. The 2018 European Heart Rhythm Association Practical Guide on the use of non-vitamin K antagonist oral anticoagulants in patients with atrial fibrillation. Eur Heart J. 2018;39(16):1330-93.

13. SAVAYSA [package insert]. Tokyo Japan DSC, LTD. 2019.

14. Lixiana [package insert]. Tokyo Japan: Daiichi Sankyo Europe GmbH.; 2021.

15. Gu JQ, Guo YP, Jiao Z, Ding JJ, Li GF. How to Handle Delayed or Missed Doses: A Population Pharmacokinetic Perspective. Eur J Drug Metab Pharmacokinet. 2020;45(2):163-72.

16. Ding JJ, Zhang YJ, Jiao Z, Wang Y. The effect of poor compliance on the pharmacokinetics of carbamazepine and its epoxide metabolite using Monte Carlo simulation. Acta Pharmacol Sin. 2012;33(11):143140.

17. Saint-Marcoux F, Woillard JB, Monchaud C, Friedl J, Bocquentin F, Essig M, et al. How to handle missed or delayed doses of tacrolimus in renal transplant recipients? A pharmacokinetic investigation. Pharmacol Res. 2015;100:281-7. 
18. MacCannell D, Berger Z, East L, Mercuri E, Kirschner J, Muntoni F, et al. Population pharmacokinetics-based recommendations for a single delayed or missed dose of nusinersen. Neuromuscul Disord. 2021;31(4):310-8.

19. Wang CY, Jiao Z, Ding JJ, Yu EQ, Zhu GX. Remedial dosing recommendations for delayed or missed doses of valproic acid in patients with epilepsy based on Monte Carlo simulations. Epilepsy Behav. 2020;111:107265.

20. Liu XQ, Yin YW, Wang CY, Li ZR, Zhu X, Jiao Z. How to handle the delayed or missed dose of rivaroxaban in patients with non-valvular atrial fibrillation: model-informed remedial dosing. Expert Rev Clin Pharmacol. 2021;14(9):1153-63.

21. Feinberg WM, Blackshear JL, Laupacis A, Kronmal R, Hart RG. Prevalence, age distribution, and gender of patients with atrial fibrillation. Analysis and implications. Arch Intern Med. 1995;155(5):469-73.

22. Hindricks G, Potpara T, Dagres N, Arbelo E, Bax JJ, Blomstrom-Lundqvist C, et al. 2020 ESC Guidelines for the diagnosis and management of atrial fibrillation developed in collaboration with the European Association for Cardio-Thoracic Surgery (EACTS): The Task Force for the diagnosis and management of atrial fibrillation of the European Society of Cardiology (ESC) Developed with the special contribution of the European Heart Rhythm Association (EHRA) of the ESC. Eur Heart J. 2021;42(5):373-498.

23. Song S, Kang D, Halim AB, Miller R. Population pharmacokinetic-pharmacodynamic modeling analysis of intrinsic FXa and bleeding from edoxaban treatment. J Clin Pharmacol. 2014;54(8):910-6.

24. Wang W, Hallow KM, James DA. A Tutorial on RxODE: Simulating Differential Equation Pharmacometric Models in R. CPT Pharmacometrics Syst Pharmacol. 2016;5(1):3-10.

25. Samuelson BT, Cuker A, Siegal DM, Crowther M, Garcia DA. Laboratory Assessment of the Anticoagulant Activity of Direct Oral Anticoagulants: A Systematic Review. Chest. 2017;151(1):127-38.

26. Samuelson BT, Cuker A. Measurement and reversal of the direct oral anticoagulants. Blood Rev. 2017;31(1):77-84.

27. Cuker A, Siegal D. Monitoring and reversal of direct oral anticoagulants. Hematology Am Soc Hematol Educ Program. 2015;2015:117-24.

28. Vranckx P, Valgimigli M, Heidbuchel H. The Significance of Drug-Drug and Drug-Food Interactions of Oral Anticoagulation. Arrhythm Electrophysiol Rev. 2018;7(1):55-61.

29. Pisters R, Lane DA, Nieuwlaat R, de Vos CB, Crijns HJ, Lip GY. A novel user-friendly score (HASBLED) to assess 1-year risk of major bleeding in patients with atrial fibrillation: the Euro Heart Survey. Chest. 2010;138(5):1093-100.

30. Lip GY, Nieuwlaat R, Pisters R, Lane DA, Crijns HJ. Refining clinical risk stratification for predicting stroke and thromboembolism in atrial fibrillation using a novel risk factor-based approach: the euro heart survey on atrial fibrillation. Chest. 2010;137(2):263-72.

\section{Hosted file}

ednoxaban tabel upload.docx available at https://authorea.com/users/444858/articles/547028how-to-handle-the-delayed-or-the-missed-dose-of-edoxaban-in-patients-with-non-valvularatrial-fibrillation-model-informed-remedial-strategy

\section{Hosted file}

figure.docx available at https://authorea.com/users/444858/articles/547028-how-to-handlethe-delayed-or-the-missed-dose-of-edoxaban-in-patients-with-non-valvular-atrialfibrillation-model-informed-remedial-strategy 